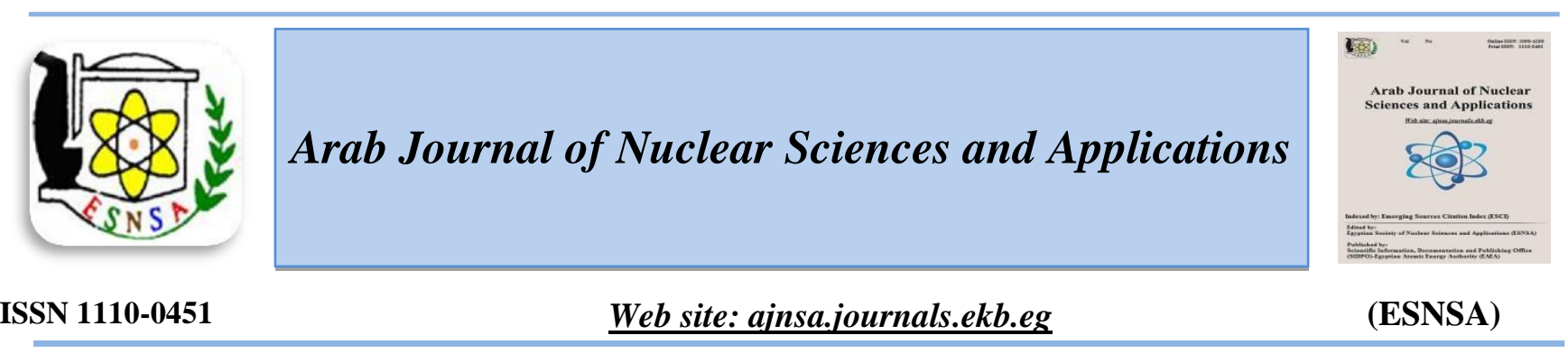

\title{
Estimation of Surface Skin Dose Using TPS and TLD of Breast Radiotherapy Using Co-60 Teletherapy Unit
}

\author{
Mohammed Elywa ${ }^{1}$, Magda Said Hanfy ${ }^{1}$ Mohamad Abd Elgawad $^{2}$ Basma El-okba $^{3}$ \\ ${ }^{1}$ Biophysics Branch, Physics Department, Faculty of Science, Zagazig University, Egypt \\ ${ }^{2}$ Nuclear medicine Department, Faculty of medicine, Zagazig University, Egypt \\ ${ }^{3}$ Physics Department, Faculty of Science, Zagazig University, Egypt
}

Received $12^{\text {th }}$ Jul. 2019 Estimation of surface skin dose is very important for patients who undergo breast radiotherapy to show Accepted $12^{\text {th }}$ Dec. 2019 that the skin dose is under the safe level and to avoid tumor recurrence. The aim of this study is utilizing the thermolumiscent dosimeters (TLDs) as a quality control tool in conventional radiotherapy procedures. Twenty patients, undergoing breast removal operations, were stimulated by treatment planning system (TPS) and six lithium floride TLD-LiF chips have been applied at the irradiated breast area. All measurements were performed using a Co-60 teletherapy (open field). All TLD chips were measured using the Harshaw 6600 reader system. The results have shown that the correlation coefficient and the Bland-Altman agreement plot of 20 patients at six points illustrated that there was no significant difference $(p>0.05)$ between TPS calculations and TLD measurements except at beams centers, where there was a highly significant difference $(p<0.001)$, when the high dose was applied. Thus, it could be concluded that not all locations in the treatment area absorbed the same dose either using TLD measurements or using TPS values

Keywords: Co-60 Radiotherapy, Breast Cancer, TLD Detectors, TPS system

\section{Introduction}

The Co-60 unit was the most admired radiotherapy used worldwide, increased in low -middle-income countries, with the ability to provide fixed energy in the $\mathrm{MeV}$ range $(1.25 \mathrm{MeV})$ of gamma rays [1]. One of the reasons to use the radiotherapy is that radiation kills cancer cells. After the radiation therapy can help to prevent cancer cells recurrence. Recurrences can take place sometimes after surgery. Therefore radiation uses to destroy remaining cancer cells. Skin dose varies noticeably over the exterior of the chest wall and depends on a number of factors, including: field size, tangential beam entry separation [2, 3], and the techniques of the treatment used [4]. Thermoluminescence is one of the processes in thermally stimulated phenomena [5] and in a general view; thermoluminescence has different applications such as radiation dosimetry. Thermoluminescent dosimeters (TLDs) measurements have been compared with the calculated dose by the treatment planning system (TPS) to evaluate the scattered dose received by thyroid [6].

Quality assurance (QA) during the treatment by radiation therapy is set to minimize unwanted exposure [7] and beam dosimetry of Co-60 teletherapy units is a necessary QA procedure, as described in the IAEA Technical Documents. In addition, one can also compare a number of measurements points with a number of different calculations at the same spatial locations. Then it is

Corresponding author: elywa2006@gmail.com

DOI: 10.21608/ajnsa.2019.14766.1234

(C) Scientific Information, Documentation and Publishing Office (SIDPO)-EAEA 
essential to statistically combine the individual deviations to make an overall quality assessment of the TPS calculation $[8,9]$.

Lithium fluoride thermoluminescent dosimeters (TLDs) were used to measure the skin received a dose, located seven TLD chips on individual patient's skin during the fraction [10-14]. The skin dose assessment is required during breast radiotherapy to guarantee that the skin dose is below the tolerance level and is adequate to prevent tumors reappearance. Conservatively, breast radiotherapy is achieved by photon beams of Co-60 [15]. In patients with grade 1, 2 breast cancer, the standard therapy is breast-conserving surgery (BCS) followed by radiotherapy to the breast tissue. Post-mastectomy radiotherapy is recommended for patients with T3, T4 tumors. Mastectomy is still an appropriate treatment for many patients with primary breast cancer.

Dose estimations from the treatment planning system (TPS) are often the only means of estimating the radiation dose reaching out-of-field locations in routine radiotherapy. However, very little data is available on the performance of these algorithms in such regions. Furthermore, TPS commissioning usually only requires data up to a few centimeters beyond the treatment field, so dose calculations at more distant regions are not supported by measured data. This work compares dose calculations from different TPS algorithms at the contralateral breast of 5 patients who underwent photon beam radiotherapy for breast cancer. The TLD data is used as a benchmark for assessing the accuracy of each algorithm [16].

The aim of this work was planned to show that the entrance skin dose assessment is essential during breast radiation therapy to comfort that the skin dose is under the patience-level and is enough to avoid tumor recurrence. The aim of the current study is to measure the skin dose using TLD technique and comparing to the treatment planning system TPS for patients have been breast surgically removed.

\section{Materials and Methods}

\section{Patients Treatment Planning}

Twenty patients aged (45 to 55 years old) who underwent CT scanning following their breast removal surgery had been examined. The prescribed dose was 50.0 Gy in twenty-five fractions for 18 patients and 40Gy in fifteen fractions for only two patients delivered by Co-60 teletherapy unit (GWXJ80, a manufactory of nuclear power institute of china).

Organs at risk (OAR) such as lungs and heart were contoured on the CT slice by the patient's oncologist. The Clinical target volume (CTV) and the planning target volume (PTV) are contoured as the final volume determined by the physician on the planning system during the planning process. It fully contains the GTV and CTV.

It is obtained as a safety margin added to the GTV/CTV to take care of the organ motion. Organs at risk (OAR) are organs adjacent to the PTV which are normal tissue, as they don't contain malignant cells. Our goal is to minimize irradiation of (OARs) as they are relatively sensitive to the ionizing radiation and if damaged, may lead to substantial morbidity.

Two tangential opposing fields were planned, locating their isocentres in the chest wall. The beam angles were adjusted to minimize the lung and heart volume irradiation. Tangential beams were designed to deliver $(95 \%-107 \%)$ of the prescribed dose to CTV volume according to ICRP [8].

\section{TLD measurements}

The skin dose of the breast was measured using lithium fluoride thermoluminescent dosimeters TLDs (TLD-100, Harshaw-Bicron, Cleveland, OH, USA) with a cross section of $4.5 \times 4.5 \mathrm{~mm}^{2}$ and thickness of $0.8 \mathrm{~mm}$ were used. The TLD-100 chips contain natural lithium (Li-7 topped with 7.5 $\%$ of Li-6). The basic dosimeter, a Harshaw TLD100 filtered in order to correct for energy response. The information of dose exposure is provided by a card reader (Harshaw 6600 TLD reader system). Superficial absorbed dose was calculated on the measurement marks as shown in figure 1 . The TLD dosimeter TLD-100 (LiF: $\mathrm{Mg}, \mathrm{Cu}, \mathrm{P}$ ) the chip with the surface area $4.5 \times 4.5 \mathrm{~mm}$ and thickness $0.8 \mathrm{~mm}$ ). Three TLD chips were used at each measurement point and placed to get the average. Six locations were used on each one of the 20 patients (Figure 1). The measured and calculated doses were compared.

\section{Planning of treatment}

A computed tomography (CT) radiotherapy simulator (Equipment Manufactory Nuclear Power Institute of China, PR China) equipped with a laser and TIGRT treatment-planning system (TPS) that was used to perform a patient virtual simulation. 


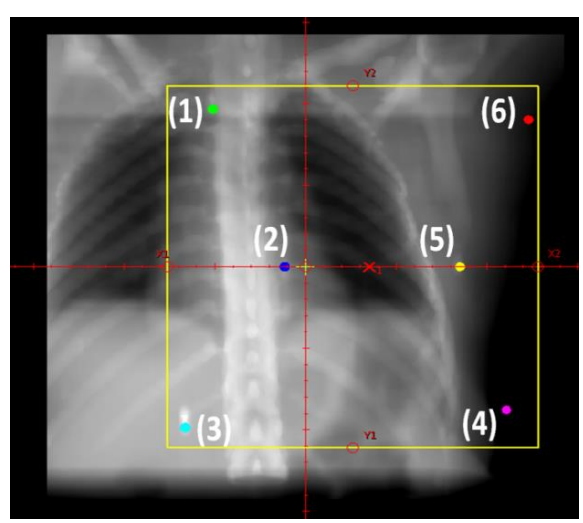

Fig. (1): shows six locations of the TLD chips. Location 1 represents the upper midpoint,- location 2 center of midpoint , location 3 lower midpoint - location 4 lower lateral point, location 5 center of lat and location 6 upper lateral point.

The images were obtained with the patients lying supine with the ipsilateral arm abducted above their heads. The scans included the entire lung in 3 mm thick -Computed tomography (CT) was used in planning the target volume. Six TLD chips located at 6 positions on the patient's chest as shown in Figure1.

Privet GWXJ80 Co-60 Teletherapy Unit (Equipment Manufactory Nuclear Power Institute of China, PR China) is used for treating breast cancer in the radiotherapy department in the governmental hospital, Egypt. It is isocentric external radiotherapy machine. The radioactive isotope is Co-60 of half live time 5 years. All patients agree on the esthetics local rules for the treatment procedures before the scanning.

\section{Statistical Analysis}

All data were acquired and analyzed using SPSS 22.0 (SPSS Inc., Chicago, IL, USA). Continuous Quantitative variables e.g. age were expressed as the mean \pm SD \& median (range), and categorical qualitative variables were expressed as absolute frequencies (number) \& relative frequencies (percentage). Continuous data were checked for normality by using the Shapiro Walk test. Wilcoxon signed ranks test was used to compare two dependent measurements of non-normally distributed data. Mann-Whitney $U$ test was used to compare two groups of non-normally distributed data. Spearman's rank correlation coefficient was calculated to assess correlations between study parameters. All tests were two-sided. P-value <
0.05 was considered statistically significant $(\mathrm{S}), \mathrm{p}-$ value $<0.001$ was considered highly statistically significant (HS), and p-value $\geq 0.05$ was considered none statistically significant (NS). We repeated the TPS and TLD measurements of skin dose in twenty subjects and using the standard Bland-Altman technique [15]. The Bland-Altman method is plotting between mean of TLD and TPS values (as the $\mathrm{X}$-value) and the difference between them (as the $\mathrm{Y}$-value), and calculates three limits 1 - the bias, the mean of difference values 2- the upper limit that is (bais+1.96*SD)and the lower limit (bais-1.96*SD) It is estimated that the 95\% limits include $95 \%$ of differences between TPS and TLD estimation methods.

Deviations between results of TPS calculations and TLD measurements can be explained as a percentage (\%) of the locally measured dose [17] and is presented that the following equation should be used:

Error $(\%)=100 \times \frac{\mathrm{D}_{\mathrm{TPS}}-\mathrm{D}_{\mathrm{TLD}}}{\mathrm{D}_{\mathrm{TLD}}}$

Where, $\mathrm{D}_{\mathrm{TLD}}$ and $\mathrm{D}_{\text {TPS }}$ are the measured doses by TLD-100 chips and the calculated dose by TPS, respectively. Finally, the difference values were obtained for in-field regions and were compared to the tolerance limit suggested in TRS 430and TECDOC 1540 protocols [18].

\section{Results and Discussion}

Correlation is a statistical skill that can indicate how strongly, TPS and TLD values are related and also p-value represents the statistical difference significant $\mathrm{s}$ between them. Table 1 indicates the mean, standard mean error, p-values and Pearson correlation coefficient ( $r$ ) of the two methods for twenty subjects at 6 points. The absolute value of correlation has been described by [19].

Figures 2 indicate the mid beam and lateral beam of Co-60 gamma rays. Breast region boundary covered by medial and lateral tangential fields in transverse view, two locations of the TLD chips: Location 1 represents the upper midpoint on the mid beam, and location 6 shows the upper lateral point on the lateral beam (Figure $2 \mathrm{~A}$ ). Location 2 lies on the center of the mid beam, and location 5 shows the center of the lateral beam, centers of mid and lateral beams (Figure 2 B). Location 3 represents the lower midpoint on the mid beam, and location 4 shows the lower lateral point on the lateral beam (Figure $2 \mathrm{C}$ ).

Results in table 1 represent that at points 2 and 5 (center mid and center lat) respectively, as shown 
in Figure $2 \mathrm{~B}$, there is a moderate positive correlation with very high significant difference $(p<0.001)$ between the collected values TPS and measured values TLD. At lower border points 3 and 4 (lower mid and lower lat) respectively, as shown in Figure $2 \mathrm{C}$, there is a strong positive correlation and there was no significant difference ( $p>0.05$ ) between TPS and TLD values. At upper border points 1 and 6 (upper mid and upper lat) respectively, as shown in Figure $2 \mathrm{~A}$, there is a very strong positive correlation at point 1 and moderate positive correlation at point 6 , there was no significant difference $(p>0.05)$ between TPS and TLD values.

The calculated TPS and that measured TLD doses described in Table 1 and Figure 3, where there is a difference in doses between TPS and TLD at all points of interesting.

Table 1 also shows that there is a positive difference between values of TPS and TLD at point $1,2,3$ and $5(25 \%, 23 \%, 13 \%$, and $22 \%)$ respectively and negative difference at point 4 and 6. This difference increased or decreased depending on the location of points on the chest and on the beam dose. The main observations that obtained from the data in Table 1 ensured by Figure 5 which shows the fit data line (red line) of twenty values of twenty subject at six locations (points). Figure 5 represents the linearity relation between TLD as X-values and TPS as Y-values and shows the fitted equation plus the r-value at each point.

The agreement between the TLD measurements and TPS calculations has been observed by using Bland-Altman plots between the mean of two variables as $\mathrm{X}$-axis and the difference as $\mathrm{Y}$-axis. Figure 6 describes the bais (mean) and the upper limit of agreement and the lower limit of agreement for twenty subjects at six points. In our study, we can describe the degree of agreement by estimation the bias (the difference between the line of the mean of difference and line of zero) by calculation of mean and the standard deviation of the difference that illustrated in Table 2.

The results reported in Table 2 can also be explained in Figure 6 where each graph shows the relation between the difference (TPS-TLD) verse the mean (TPS+TLD) /2, and represent the lines of bais, upper and lower limits of agreement. Depending on the bais value on can explain the agreement between TPS and TLD measurements whereas bais close as zero as good agreement results in.

Since the difference means TPS-TLD, thus the sign of mean values in the table refers to which value is greater than another, at point 4 the negative means that TLD values are higher than TPS values but the bais is 0.061 that refer to high significant agreement between TLD and TPS measurements. Add to this, at point 6 the mean is negative and the bais is 0.249 that still represents the signed agreement. On the other hand at point 2 and 5 the mean is positive but there are no significant agreements. In addition, at point 1,3 the mean is positive that mean TPS values are greater than TLD values but the bais is very small and indicated that high significant agreement between two measurements.

The important parameter in estimation the dose received by a patient skin is the ESD during the exposure time. ESD as a physical quantity has been identified by the European Union to be controlled as a diagnostic indication level of optimizing patient dose [20,21]. Even the medical physicists hope to use this parameter as a standard method to verify the accuracy of using TLD as accurate measurements, but that it is difficult because should be taken into account more dissimilar data [22]. TLD chips have been used to estimate the Entrance Skin Dose (ESD), absorbed dose by skin, at six locations on the chests of the patient who undergo breast surgery. The Australian Radiation Protection and Nuclear Safety Agency [23] reported recommendations of ESD values for an adult of average weight $(70-80 \mathrm{~kg})$.

Our results illustrate that there is no significant difference between measured and calculated doses of breast achieved as part of quality control and matched with [24]. The statistical analysis shows that the skin dose received points 2 and 5 at beams center of the treatment region was more than that received by the other points $1,3,4$ and 6 at the corners ( $\mathrm{p}<0.001)$. This result may be caused by the different entrance doses of the lateral and medial beams since the skin dose from the entry beam related to the beam's incident angle $[25,26]$. Our results depending on the real measured TLD and acquired TPS values investigated that TLD ships calibrated and modified can be used as a controlled dose evaluation relative to TPS measurement. This appears in the discussion of Tables 1, 2 and in Figures 5 and 6, at all points except at the center of medial and lateral beams. 


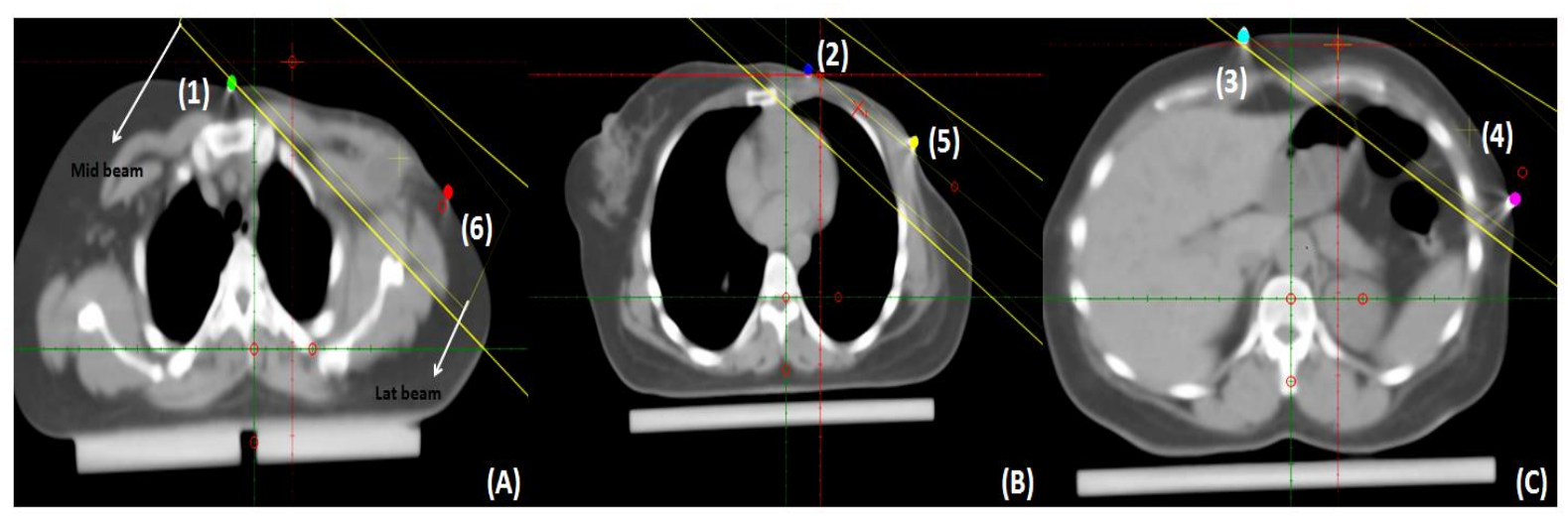

Fig. (2): Breast region boundary covered by medial and lateral tangential fields in transverse view. A) Location 1and 6 represent the upper midpoint on the mid beam and the upper lateral point on the lateral beam respectively. B) Location 2and 5 lies on the center of the mid beam and the center of the lateral beam respectively. C) Location 3 and 4 describe the lower midpoint on the mid beam and the lower lateral point on the lateral beam respectively.

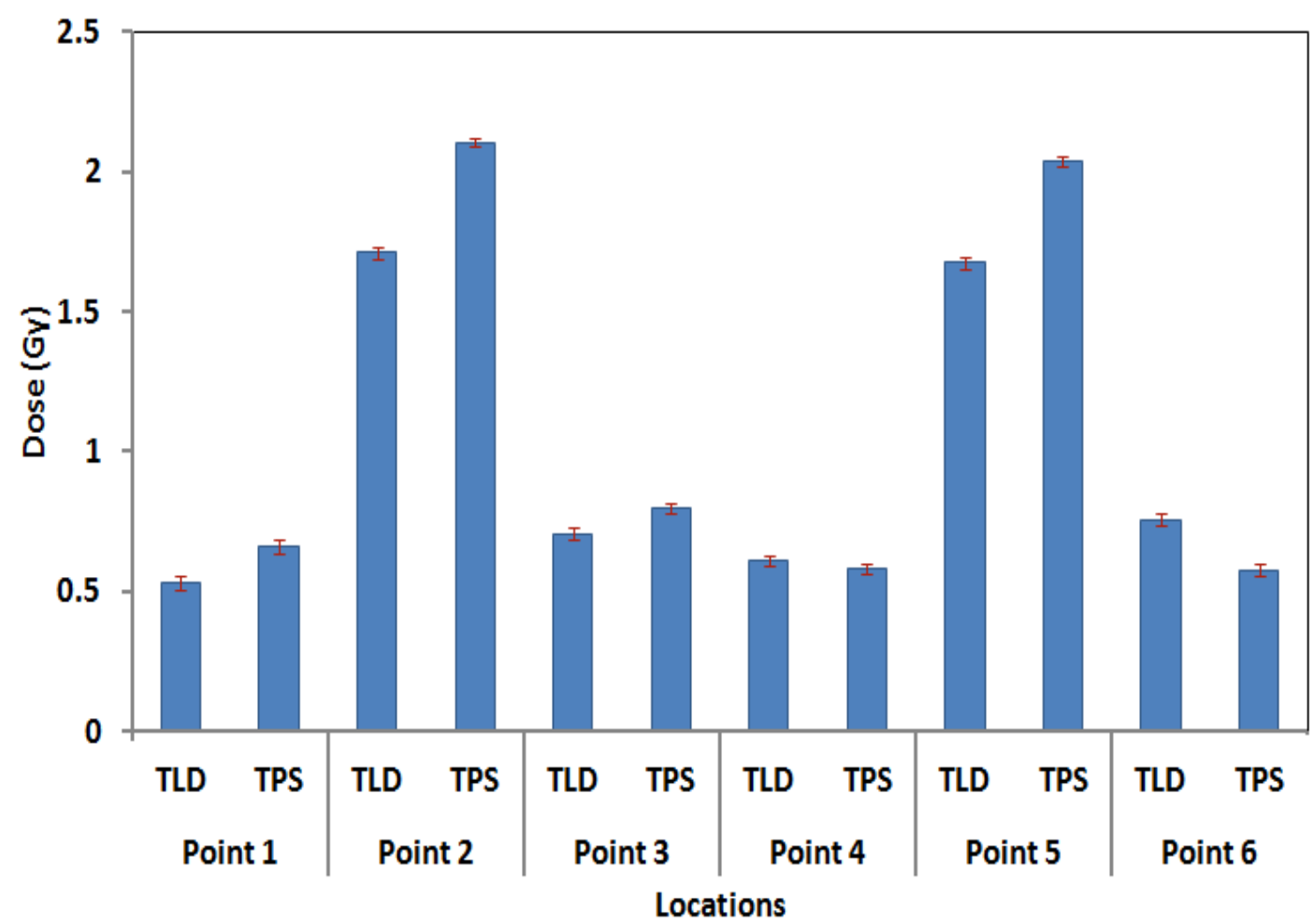

Fig. (3): shows the entrance skin dose mean \pm uncertainty that measured (TLD) and calculated (TPS) at six points of the treated chest area 
Table (1): Mean, standard deviation, TPS-TLD difference, standard mean error (SME), error (\%), p-value and correlation coefficient $(r)$ and the correlation strength of twenty subjects at 6 points

\begin{tabular}{|c|c|c|c|c|c|c|c|c|}
\hline & & Mean & SD & SME & Error (\%) & $\begin{array}{c}\text { p- } \\
\text { value }\end{array}$ & $\mathbf{r}$-value & $\begin{array}{c}\text { Positive } \\
\text { correlation [19] }\end{array}$ \\
\hline \multirow{2}{*}{$\begin{array}{l}\text { Point } 1 \\
\text { Upper mid }\end{array}$} & TLD & 0.531 & 0.448 & 0.0224 & \multirow[b]{2}{*}{$25 \%$} & \multirow[b]{2}{*}{0.0661} & \multirow[b]{2}{*}{0.85} & \multirow[b]{2}{*}{ Very strong } \\
\hline & TPS & 0.664 & 0.505 & 0.0253 & & & & \\
\hline \multirow{2}{*}{$\begin{array}{l}\text { Point } 2 \\
\text { Center mid }\end{array}$} & TLD & 1.709 & 0.453 & 0.0227 & \multirow{2}{*}{$23 \%$} & \multirow{2}{*}{0.0007} & \multirow{2}{*}{0.41} & \multirow{2}{*}{ Moderate } \\
\hline & TPS & 2.103 & 0.328 & 0.0164 & & & & \\
\hline \multirow{2}{*}{$\begin{array}{c}\text { Point } 3 \\
\text { Lower mid }\end{array}$} & TLD & 0.706 & 0.409 & 0.0205 & \multirow{2}{*}{$13 \%$} & \multirow{2}{*}{0.2512} & \multirow{2}{*}{0.69} & \multirow{2}{*}{ Strong } \\
\hline & TPS & 0.797 & 0.416 & 0.0208 & & & & \\
\hline \multirow{2}{*}{$\begin{array}{c}\text { Point } 4 \\
\text { Lower lat }\end{array}$} & TLD & 0.607 & 0.358 & 0.0179 & \multirow{2}{*}{$-4 \%$} & \multirow{2}{*}{0.6933} & \multirow{2}{*}{0.72} & \multirow{2}{*}{ Strong } \\
\hline & TPS & 0.581 & 0.407 & 0.0204 & & & & \\
\hline \multirow{2}{*}{$\begin{array}{l}\text { Point } 5 \\
\text { Center lat }\end{array}$} & TLD & 1.673 & 0.372 & 0.0186 & \multirow{2}{*}{$22 \%$} & \multirow{2}{*}{0.0003} & \multirow{2}{*}{0.44} & \multirow{2}{*}{ Moderate } \\
\hline & TPS & 2.035 & 0.323 & 0.0162 & & & & \\
\hline \multirow{2}{*}{$\begin{array}{l}\text { Point } 6 \\
\text { Upper lat }\end{array}$} & TLD & 0.757 & 0.426 & 0.0213 & \multirow{2}{*}{$-24 \%$} & \multirow{2}{*}{0.0794} & \multirow{2}{*}{0.58} & \multirow{2}{*}{ Moderate } \\
\hline & TPS & 0.577 & 0.417 & 0.0209 & & & & \\
\hline
\end{tabular}

$p>0.05$ non significant; $P \leq 0.05$ : significant; $p<0.000$ very high significant
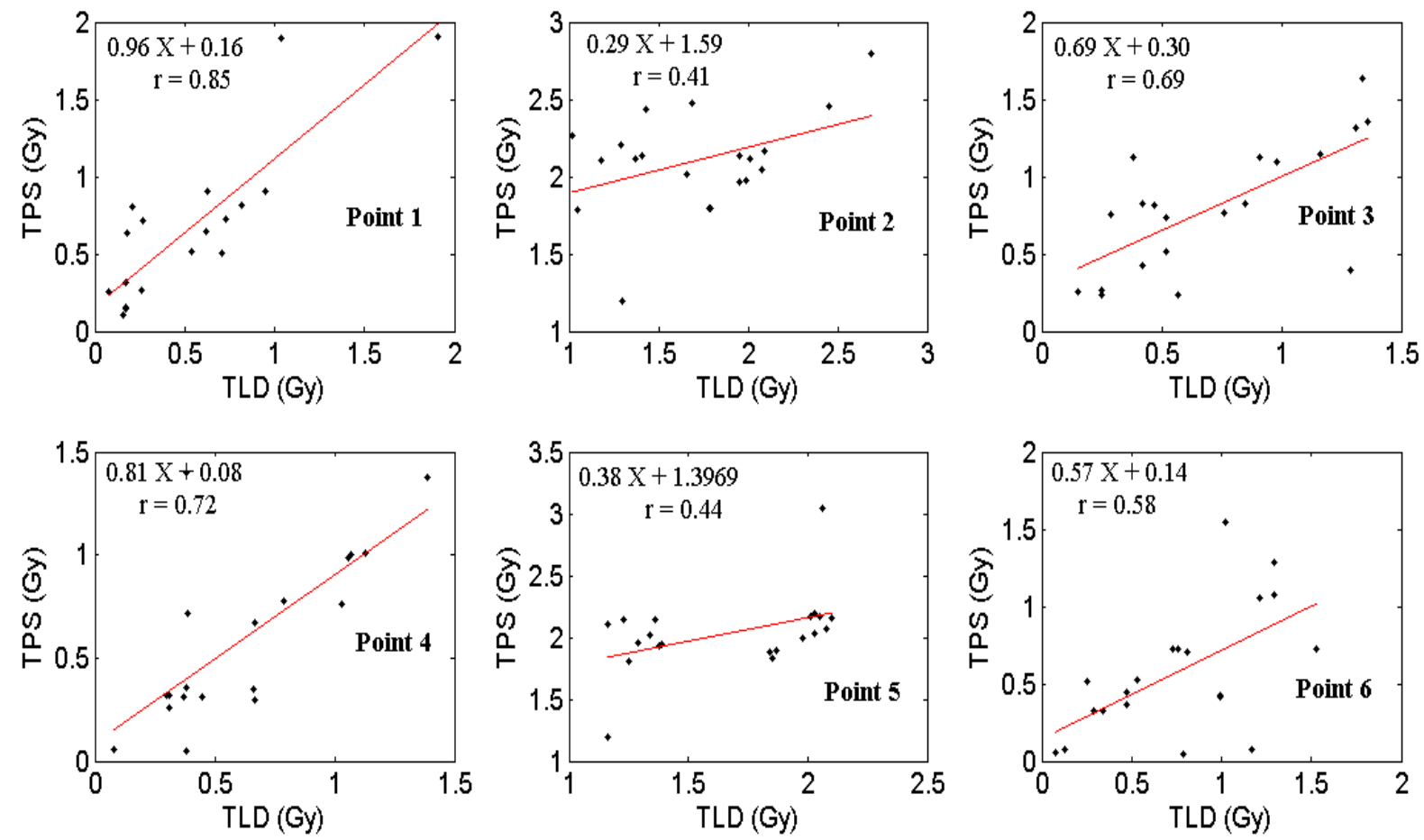

Fig. (4): scatter plots between TLD and TPS values of twenty patients at six locations. At points 1 and 4 , there is a very strong positive relationship between TLD measurements and TPs values. At points 2 and 5, there is a very weak positive relation between TLD measurements and TPs values. At points 3 and 6 , there is a moderate positive relation between TLD measurements and TPS values. 
Table (2): The mean of difference, standard deviation (SD) of the difference, lower limit of agreement (mean$1.96 * \mathrm{SD})$ and upper limit of agreement (mean+1.96*SD)

\begin{tabular}{ccccc}
\hline & Mean (Gy) & SD(Gy) & The upper limit(Gy) & The lower limit(Gy) \\
\hline Point 1 & 0.182 & 0.249 & 0.671 & -0.308 \\
Point 2 & 0.724 & 0.313 & 1.338 & 0.109 \\
Point 3 & 0.189 & 0.380 & 0.935 & -0.555 \\
Point 4 & -0.061 & 0.305 & 0.536 & -0.658 \\
Point 5 & 0.414 & 0.481 & 1.356 & -0.528 \\
Point 6 & -0.249 & 0.418 & 0.571 & -1.068 \\
\hline
\end{tabular}
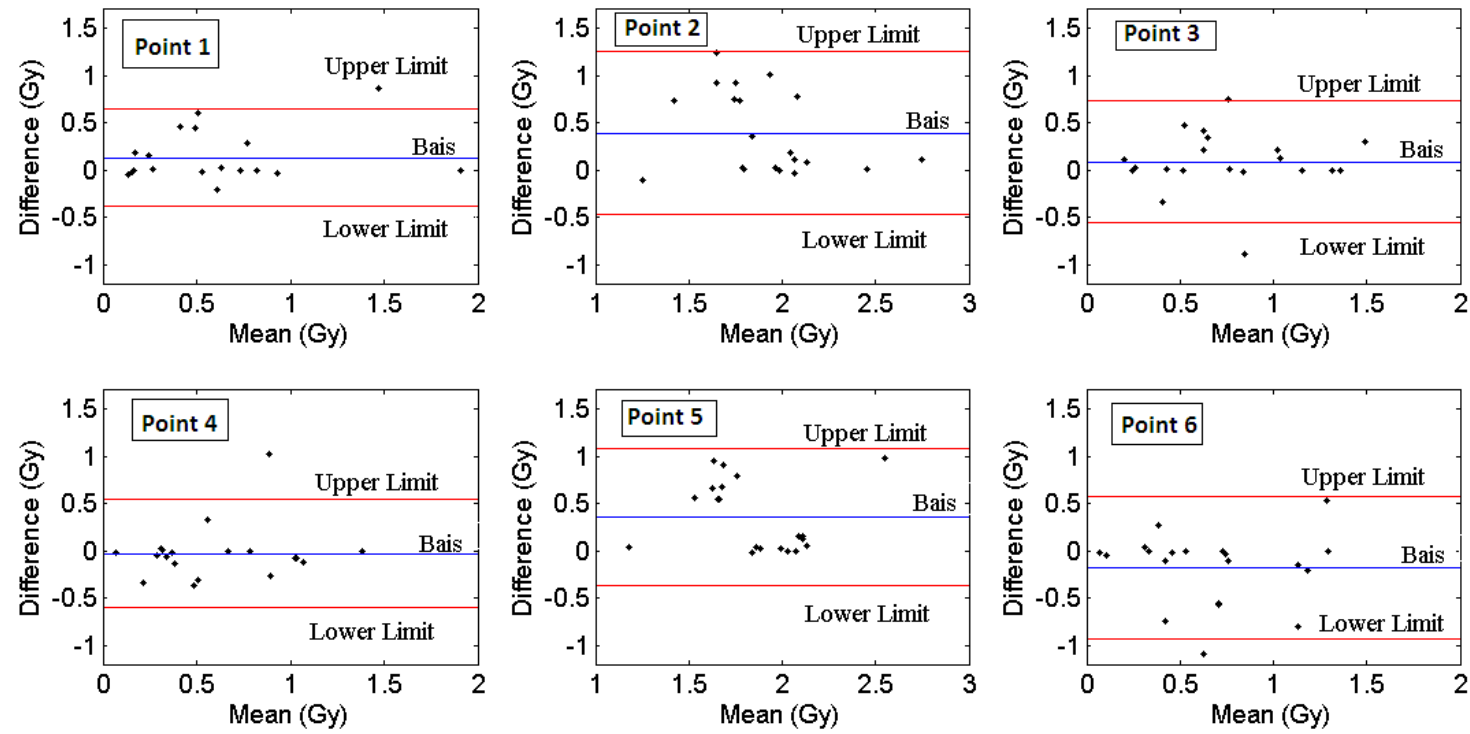

Figure 5 Bland-Altman plot of 20 patients. The difference between TLD and TPS is drawn against the mean of TLD and TPS in the twenty measurements in the study at 6 points. The smaller the range between the upper limit and lower limit, the better the agreement between the TLD and TPS.

\section{Conclusion}

Based on the data reported in this study, the skin dose of the breast at the center of beams is higher than at corners, also the correlation coefficient and the Bland-Altman plot of 20 patients at six locations illustrate that no significant difference between TPS and TLD measurement except at the center of beams. Where there is a small difference between mean values of TPS and TLD at point 1 and 3, which maybe because of the two-point lie on the tangents of the beam boundaries. This difference increased or decreased depending on the dimension of point in the beam field. The difference becomes very high at points 2 and 5 , i.e., at the center of beams.

\section{Aknologement}

We would like to thank Dr. Emad Moustafa and all the members of Facus Radiotherapy Center, Sharkia, Egypt.

\section{References}

1. Schreiner LJ. Dosimetry in modern radiation therapy: limitations and needs. J. of Phys. Conference Series. 2006; 56: 1-13.

2. Khan FM. The physics of radiation therapy. 4th edition. Lippincott Williams \& Wilkins, Philadelphia; 2010. 
3. Pires AM, Segreto RA, Segreto HR. RTOG criteria to evaluate acute skin reaction and its risk factors in patients with breast cancer submitted to radiotherapy. Rev Lat Am Enfermagem 2008; 16: 844-9.

4. Kelly A, Hardcastle N, Metcalfe P, et al. Surface dosimetry for breast radiotherapy in the presence of immobilization cast material. Phys Med Biol. 2011; 56: 1001-13.

5. McKeever, S., Moscovitch, M., Townsend, P. Thermoluminescence Dosimetry Materials: Properties and Uses. Nuclear Technology Publishing, England.1995

6. Senthilkumar, S. Design of homogeneous and heterogeneous human equivalent thorax phantom for tissue inhomogeneity dose correction using TLD and TPS measurements. International Journal of Radiation Research. 2014; 12 (2): 169-178

7. IAEA. Commissioning and quality assurance of computerized planning systems for radiation treatment of cancer. Technical report series 430. Vienna: IAEA; 2004.

8. IAEA. Design and implementation of a radiotherapy programme: Clinical, medical physics, radiation protection, and safety aspects. TECDOC-1040. Vienna: IAEA; 1996.

9. International Atomic Energy Agency. Quality assurance in radiotherapy. TECDOC-989. Vienna: IAEA; 1997.

10. Shokouhozaman S., Aledavood S. A., Noghreiyan A. V., Ghorbani M., Jamali F. and Davenport D. In vivo skin dose measurement in breast conformal radiotherapy. Contemp Oncol (Pozn). 2016; 20: 137-140

11. Sari F., Mahdavi S. R., Anbiaee R., Shirazi A. The Effect of Breast Reconstruction Prosthesis on Photon Dose Distribution in Breast Cancer Radiotherapy.Iranian Journal of Medical Physics,2017;14 (4): 251-256

12. Bouzarjomehri F., and Yazdi M. R. A comparison of contralateral breast dose due to breast cancer radiotherapy using two different treatment machines in a radiotherapy center. International Journal of Radiation Research, 2017; 15(3):295-299

13. Mahdavi1 S. R., Tutuni1M., Farhood B., Nafisi N., Ghasemi S., Mirzaee H., Ahmadi S. and Alizadeh A. Measurement of peripheral dose to the pelvic region and the associated risk for cancer development after breast intraoperative electron radiation therapy. J. Radiol. Prot. 2019; 39: 278-291
14. Soleymanifard S., Aledavood S. A., Noghreiyan A. V., Ghorbani M., Jamali F. and Davenport D. In vivo skin dose measurement in breast conformal radiotherapy. Contemp. Oncol. 2016; 20: 137-40,

15. Bland JM, Altman DG. Statistical methods for assessing agreement between two methods of clinical measurement. Lancet. 1986; 307-310

16. Howell RM, Scarboro SB, Taddei PJ, Krishnan S, Kry SF, Newhauser WD. Methodology for determining doses to in-field, out-of-field and partially in-field organs for late effects studies in photon radiotherapy. Phys Med Biol. 2010;55(23):7009-7023.

17. VENSELAAR, J., WELLEWEERD, H., MIJNHEER, B., Tolerances for the accuracy of photon beam dose calculations of treatment planning systems, Radiother. Oncol. 2001; 60: 191-201.

18. Vatnitsky, S. Specification and acceptance testing of radiotherapy treatment planning systems. Vienna: IAEA. 2007

19. Evans, J. D. Straightforward statistics for the behavioral sciences. Belmont, CA, US: Thomson Brooks/Cole Publishing Co. 1996.

20. European Commission. European guidelines on quality criteria for diagnostic radiographic images. EUR 16260 EN. Brussels; 1996.

21. Muhogora WE, Nyanda AM, Kazema RR. Experiences with the European guidelines on quality criteria for radiographic images in Tanzania. J Appl Clin Med Phys. 2001;2(4):219-226.

22. Compagnone G., Pagan L., and Bergamini C. Comparison of six phantoms for entrance skin dose evaluation in 11 standard X-ray examinations.Journal Of Applied Clinical Medical Physics, 2005; 6(1):101-13

23. Basic Physics of Digital Radiography/The Patient - Wikibooks, open books for an open world". En.wikibooks.org, 2017.

24. Evwierhurhoma OB, Ibitoye ZA, Ojieh CA, and Duncan JTK, Ann Med Health Sci Res. 2015; 5(6): 409-12.

25. Quach K, Morales J, Butson M, Rosenfeld AB, Metcalfe PE. Measurement of radiotherapy Xray skin dose on a chest wall phantom. Med Phys 2000; 27: 1676-80.

26. Cheung T, Butson MJ, Yu PK. Multilayer Gafchromic film detectors for breast skin dose determination in vivo. Phys Med Biol 2002; 47: 31-7. 